\title{
High Selectivity Wideband BPF With Very Wide Stopband Characteristic
}

\author{
Muhammad Riaz ${ }^{1}$, Bal S. Virdee ${ }^{2}$, Mohammad Alibakhshikenari ${ }^{3}$, Louis Wy Liu ${ }^{4}$ \\ ${ }^{1}$ Dept. of Electrical Engineering, Grand Charter College of Engineering and Technology, Lahore, Pakistan. \\ ${ }^{2}$ Centre for Communications Technology, School of Computing \& Digital Media, London Metropolitan University, UK \\ ${ }^{3}$ Electronic Engineering Department, University of Rome "Tor Vergata", Via del Politecnico 1, 00133, Rome, Italy \\ ${ }^{4}$ EEIT Department, Vietnamese German University, Thu Dau Mot City, Binh Duong Province, Vietnam
}

Corresponding Author: Bal S. Virdee

\begin{abstract}
This paper describes the design and evaluation of a highly selective wideband microstrip bandpass filter with a near brick-shaped transmission response and a very wide stopband characteristic. The proposed filter structure excites multimode resonances that combine to realize a wideband filter response and excited too are transmission zeros that create a highly selective filter with wideband suppression in the upper and lower stopbands. The filter configuration comprises electromagnetically coupled resonators that are stub loaded. The input and output feedlines are interdigitally coupled to the resonators. Measured results confirm the low-loss and via-free wideband filter exhibits an elliptical response with a wide stopband with a rejection greater than $30 \mathrm{~dB}$. The selectivity factor and stopband performance of the proposed filter is better than that obtained with the hightemperature superconductor (HTS) filters. Design of the filter is relatively simple and easy to manufacture using standard PCB technology. There is good correlation between the simulation and measured results. The proposed wideband bandpass filter is suitable for applications in high interference environments and cognitive radio systems.
\end{abstract}

Index Terms-Planar Filters, microstrip technology, wide stopband, transmission-line structure.

\section{INTRODUCTION}

$\mathrm{N}$ OWADAYS the use of wideband systems has become common for various applications such as high-precision range-measurement radars, anti-interference and electronic countermeasure systems, and high-data rate short distance wireless communication systems. Frequency synthesizer is an integral component of such systems that needs to support broadband operation. Therefore, it is important to suppress the spurs and harmonics generated in the frequency synthesizer in order to guarantee the purity of its output signal. Although we can optimize the design of the frequency synthesizer however it's equally important to utilize wideband bandpass filters with high-selectivity and wide stopband to ensure the performance of the synthesizer.

Recently, several wideband filters have been developed [1][5]. In [1] the wideband filter design uses three-mode dual-ring resonator, where the resonator is constructed from two quarter wavelength transmission-lines and a cascaded half wavelength dual-ring. The bandwidth of this filter is controlled by controlling the positions of the transmission zeros produced by the resonator. The measured response of the filter agrees well with the design simulation. The fabricated filter has an insertion-loss which of better than $1.5 \mathrm{~dB}$ in the passband, and the two rejection responses are greater than $20 \mathrm{~dB}$ in the stopbands between 0 to $10 \mathrm{GHz}$. In [2] a wideband bandpass filter design is based on two dual mode stub-loaded steppedimpedance resonators. Two electric coupling paths and one common magnetic coupling path are introduced between the two resonators to produce multiple transmission zeros at both sides of the passband, resulting in high passband selectivity. However, the filter design requires two via-holes that complicate its fabrication. This four-pole wideband bandpass filter which is centered at $3.35 \mathrm{GHz}$ and having a $3-\mathrm{dB}$ fractional bandwidth of $32.96 \%$, has a measured insertion-loss of $0.95 \mathrm{~dB}$ and return-loss is better than $11 \mathrm{~dB}$. In [3] the wideband bandpass filter consists of balanced microstrip slotline transition structures and two sets of nested split-ring resonators. The resonators excite a wide differential mode passband centered at $3.0 \mathrm{GHz}$. The structure generates two transmission zeros to provide high frequency selectivity. This filter has a $3-\mathrm{dB}$ fractional bandwidth of $28.3 \%$ and insertionloss of $0.91 \mathrm{~dB}$. In [4] a wideband bandpass filter is based on folded substrate integrated waveguide (SIW), where five square complementary split-ring resonators are etched in the middle layer of the waveguide. The resonant frequency of the resonators and the filter's stopband performance can be changed by adjusting the physical size of the resonators. To enhance the impedance matching characteristics of the filter, two microstrip-lines to stripline transitions are added in two ports of the filter. The measured results show the filter has an insertion-loss of $1.2 \mathrm{~dB}$ and a fractional bandwidth of $35.5 \%$. In [5] the wideband filter is implemented using crossover coupled lines. The dual-mode filter is designed centered at 2.03 and 3.54 $\mathrm{GHz}$ with measured bandwidths of 0.6 and $0.62 \mathrm{GHz}$, respectively. At $2.03 \mathrm{GHz}$ the filter has an insertion-loss of 1 $\mathrm{dB}$ and return-loss of $18 \mathrm{~dB}$, and at $3.54 \mathrm{GHz}$ the insertion-loss is $1.2 \mathrm{~dB}$ and return-loss of $22 \mathrm{~dB}$. Out-of-band suppression of the lower and upper stopbands are better than $45 \mathrm{~dB}$. Although, the aforementioned filters are compact devices however their selectivity factor is low, insertion-loss is relatively high, and more importantly their stopband frequency range is limited.

In this paper, a compact and highly selective wideband bandpass filter is presented. The proposed filter is implemented of a planar structure and it is free of vias and its ground-plane 
requires no defection. The filter consists of electrically coupled stub-loaded multimode resonators that are electromagnetically coupled to interdigital feedlines to minimize passband insertion-loss and suppress the transmission of high order modes. The resulting filter has a quasi-elliptic function bandpass characteristic with a low passband insertion-loss, steep skirt selectivity, high rejection level on both sides of the passband over a very wide frequency span. These features are normally observed in cavity and high-temperature superconductor filters (HTS).

\section{WidEBAND MicrostriP BANDPASS FILTER CONFIGURATION}

Configuration of the proposed wideband bandpass filter, shown in Fig. 1(a), consists of two U-shaped resonators with asymmetric feedlines tapped at the center. The filter is electromagnetically excited through interdigital capacitors. The input and output feedlines divide the resonators into two sections of unequal lengths. The total length of the U-shaped resonators is $l=l_{x}+l_{y}=\lambda_{g} / 2$, where $\lambda_{g}$ is the guided wavelength at fundamental resonance. The two U-shaped resonators are partially parallel coupled to each other at the open-circuit sides, and the resonators are loaded with open-circuited stubs. We can determine the transmission zeros that define the filter's bandwidth response by analyzing the structure. To simplify the analysis the two open-circuited stub loadings are ignored. The insertion-loss of the circuit can be determined from the $Y$ parameters, thus

$$
\begin{aligned}
& S_{21}=\frac{-2 Y_{21} Y_{o}}{\left(Y_{11}+Y_{o}\right)\left(Y_{22}+Y_{o}\right)-Y_{12} Y_{21}} \\
& =\frac{j 4\left(Z_{o} \sin \beta l-\frac{\cos \beta l_{x} \cos \beta l_{y}}{\omega C_{s 1}}\right) Y_{o}}{\left[2 \cos \beta l+\frac{Y_{o} \sin \beta l}{\omega C_{s 1}}+j\left(Z_{o} \sin \beta l-\frac{\cos \beta l_{x} \cos \beta l_{y}}{\omega C_{s 1}}\right) Y_{o}\right]^{2}-4}
\end{aligned}
$$

The transmission zeros can be determined by letting $S_{21}=0$, i.e.

$$
Z_{o} \sin \beta l-\frac{\cos \beta l_{x} \cos \beta l_{y}}{\omega C_{s 1}}=0
$$

For a weak electromagnetic coupling $C_{s l}$ is small, then Eqn. (2) can be approximated to

$$
\cos \beta l_{x} \cos \beta l_{y}=0
$$

where $\beta=\left(2 \pi f \sqrt{\varepsilon_{e f f}}\right) / c, \quad \varepsilon_{\text {eff }}$ is the effective dielectric constant and $c$ is the speed of light in free-space. The frequencies of the upper $\left(f_{1}\right)$ and lower $\left(f_{2}\right)$ transmission zeros that characterize the bandpass response are determined by the lengths of $l_{y}$ and $l_{x}$, respectively, which are given by

$$
f_{1}=n c / 4 l_{x} \sqrt{\varepsilon_{e f f}} \text { and } f_{2}=n c / 4 l_{y} \sqrt{\varepsilon_{e f f}} \quad n=1,3,5 \ldots
$$

where $n$ is the mode number.
The passband of the filter is constituted from the four resonant peaks $\left(f_{e l}, f_{o 1}, f_{e 2}, f_{o 2}\right)$, shown in Fig. 1(b), that are distributed within the passband from $6.5 \mathrm{GHz}$ to $9.8 \mathrm{GHz}$. The two transmission zeros at the lower and upper cutoff frequencies provide a steep skirt selectivity.

Fig. 2 shows the stub-loaded resonator that is analyzed to determine its effect on the filter response. The resonator $\left(Z_{1}, \theta_{1}\right.$, $Z_{3}, \theta_{3}$ and $\left.Z_{6}, \theta_{6}\right)$ is loaded with open-circuited stub $\left(Z_{2}, \theta_{2}\right)$ and feedline $\left(Z_{4}, \theta_{4}\right.$ and $\left.Z_{5} \theta_{5}\right)$. Parameters $Z$ and $\theta$ represent the characteristic impedance and electrical length, respectively. It can be shown that the admittance at the junction points are given by

$$
\begin{aligned}
& Y_{r 1}=\mathrm{j} \tan \theta_{1} / Z_{1} \\
& Y_{r 2}=\mathrm{j} \tan \theta_{2} / Z_{2} \\
& Y_{x}=j\left(Z_{2} \tan \theta_{1}+Z_{1} \tan \theta_{2}\right) / Z_{1} Z_{2} \\
& Y_{r 4}=-j\left[\frac{Z_{4}+Z_{5} \tan \theta_{4} \cot \theta_{5}}{Z_{4}\left(Z_{4} \tan \theta_{4}-Z_{5} \cot \theta_{5}\right)}\right] \\
& Y_{y}=-j\left[\frac{Z_{3}+Z_{x} \tan \theta_{3} \cot \theta_{x}}{Z_{3}\left(Z_{3} \tan \theta_{3}-Z_{x} \cot \theta_{x}\right)}\right] \\
& Y_{r 5}=\mathrm{j} \tan \theta_{6} / Z_{6}
\end{aligned}
$$

The impedance $Z_{1}=Z_{3}=Z_{6}$

The resonance condition is when $Y=0$. It is evident from Eqns. (5)-(10) that structure will generate four resonant peaks as shown in Fig. 1(b). The unique feature of the proposed filter structure is its ability to suppress unwanted spurious passband responses in the upper and lower sides of the passband as is evident in Fig. 1(c). The bandpass response can be controlled by changing the length and/or the width of the open-circuited stubs.

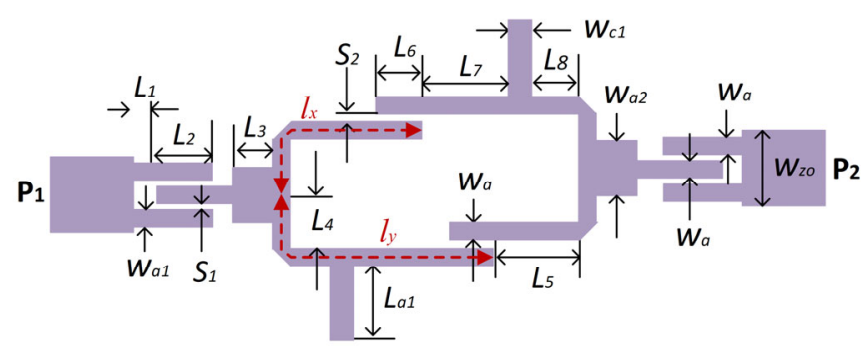

(a)

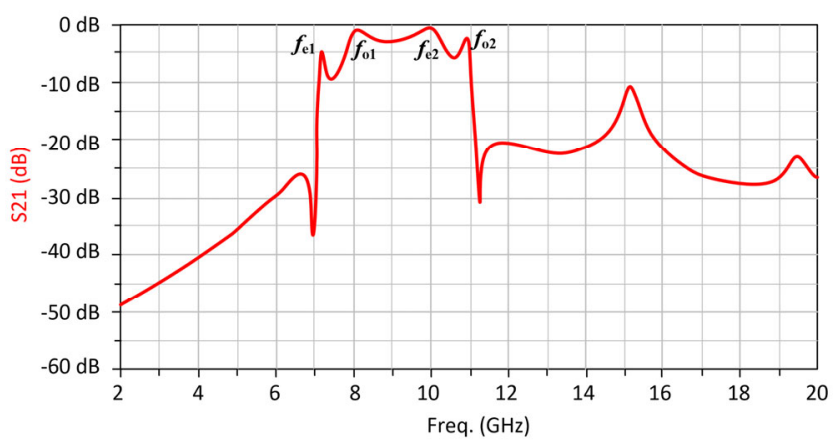

(b) 


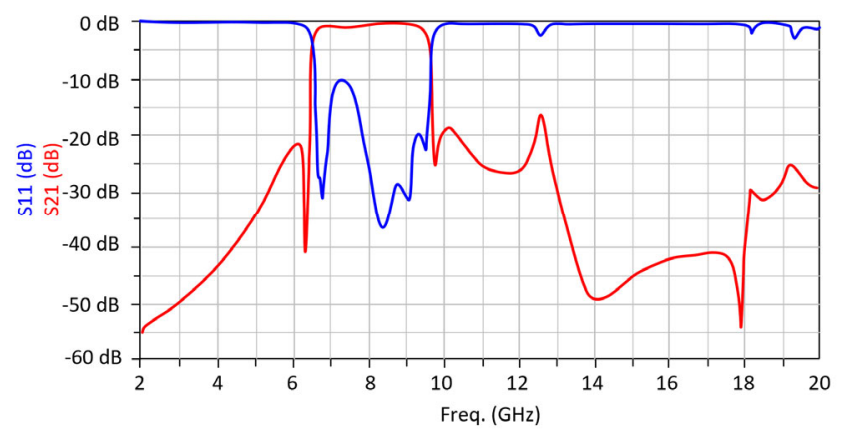

(c)

Fig. 1(a) The physical layout structure of wideband BPF filter, where $\mathrm{P}_{1}$ and $\mathrm{P}_{2}$ are input and output ports; (b) simulated unoptimized insertion-loss response under weak inter-resonator coupling; and (c) simulated unoptimized insertionloss and return-loss response under strong inter-resonator coupling.

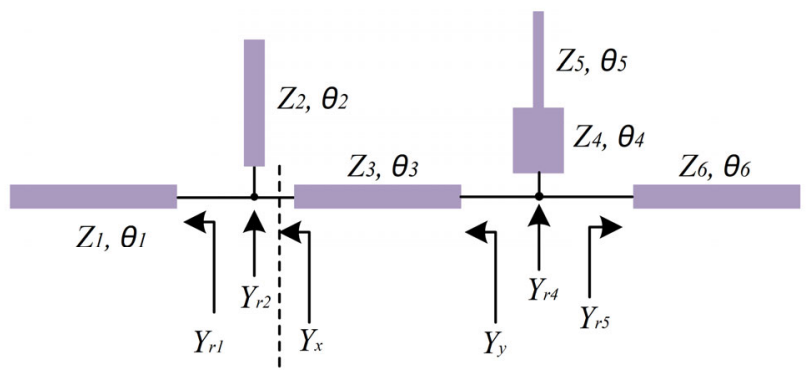

Fig. 2. Structure of the stub loaded resonator.

Simulation analysis in Fig. 3 shows the salient parameters that affect the filter's passband response. The lengths $L_{4}$ to $L_{8}$ control the frequency of the four passband resonant modes and the two transmission zeros that determine the filter's selectivity. Lengths of $L_{4}$ to $L_{6}$ are shown to control the 3-dB bandwidth of the filter. In fact, the length of $L_{5}$ has a more pronounced effect on the bandwidth. The increase in length of $L_{7}$ essentially shifts the filter's passband lower in frequency as does $L_{8}$, which is shown in Fig. 4. In the configuration the open-circuited stubs exhibit band notch functionality, and the length of the stubs $\left(\lambda_{g} / 4\right)$ was chosen to suppress the dominant out-of-band spurious response.

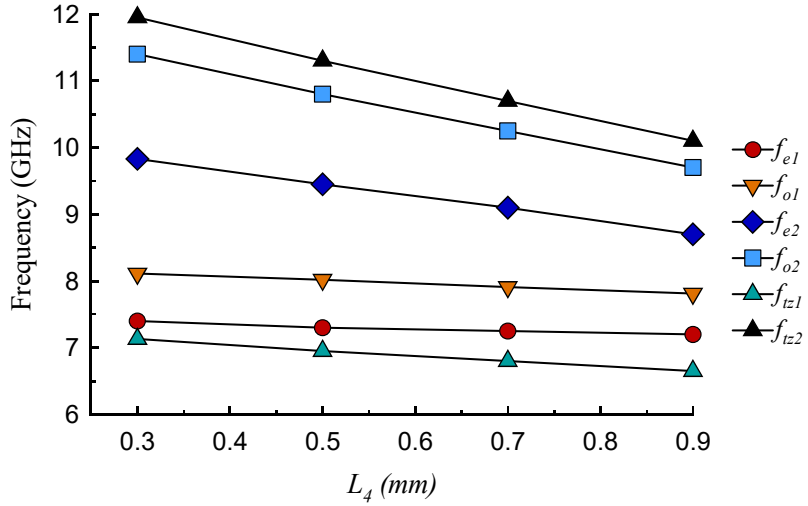

(a)

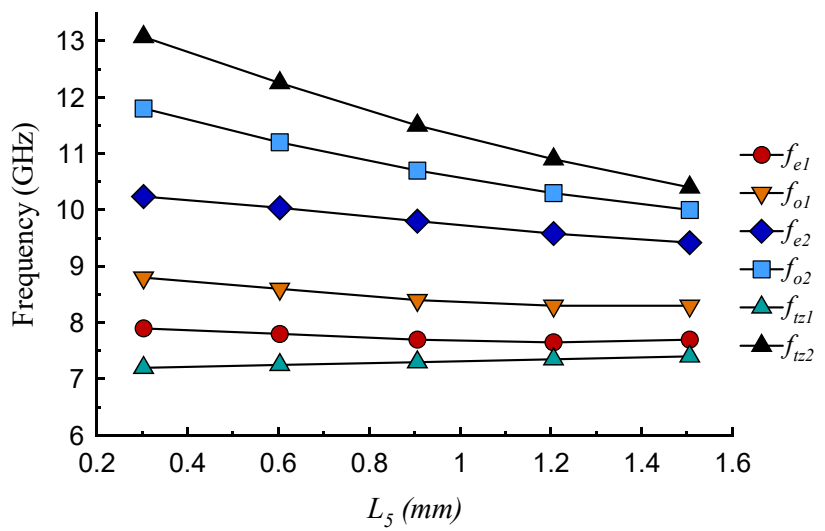

(b)

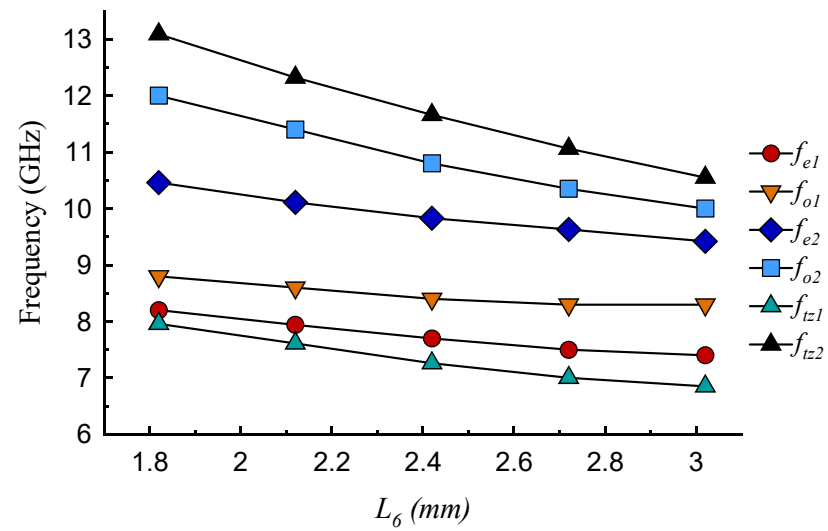

(c) 


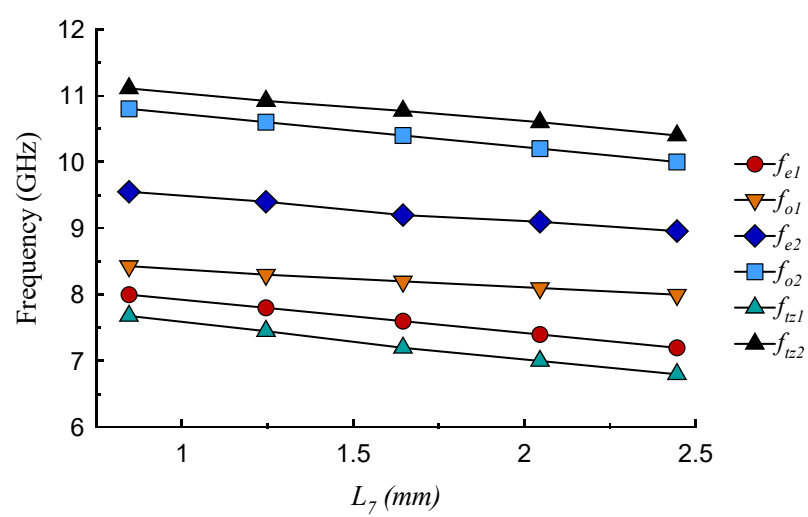

(d)

Fig. 3. Effect of salient parameters on the filter's even and odd resonant modes, and on the upper and lower passband transmission zero frequencies.

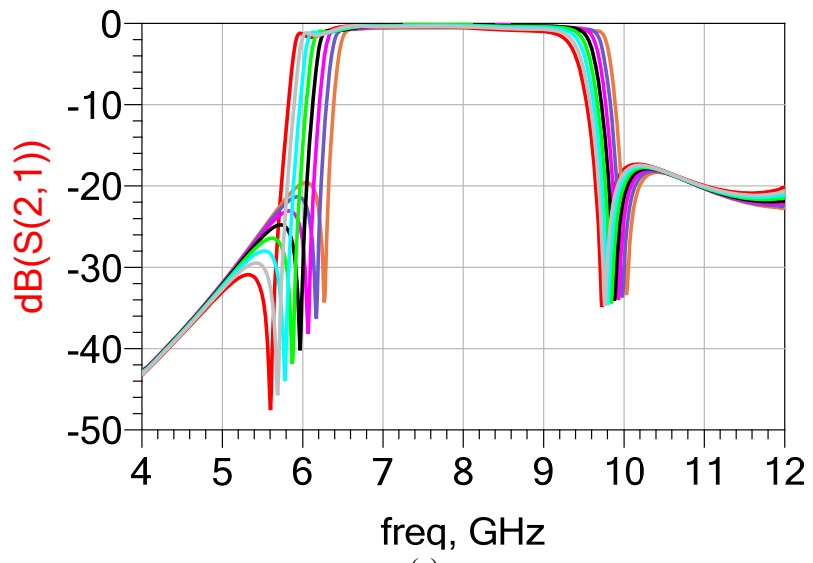

(a)

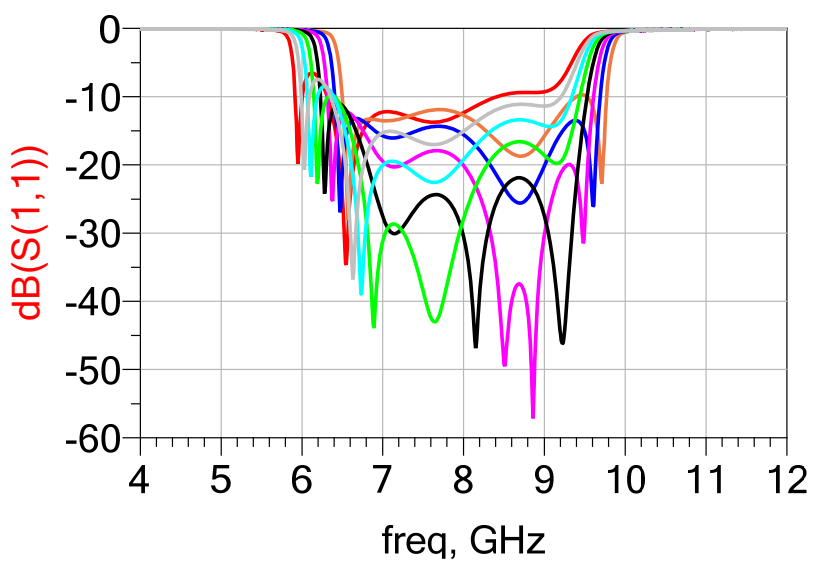

(b)

Fig. 4. Effect of length $\left(L_{8}\right)$ on the filter's insertion-loss and return-loss response when the length is varied from $1.2 \mathrm{~mm}$ to $2.4 \mathrm{~mm}$.

\section{WIDEBAND MICROSTRIP BANDPASS FILTER FABRICATION AND RESULTS}

For a given center frequency and $3-\mathrm{dB}$ bandwidth the corresponding electrical lengths constituting the filter structure in Fig. 2 can be calculated from Eqns. (1)-(6). Optimization of the design is necessary to improve the out-of-band rejection.
Simulation of the filter was carried out by using full-wave EM simulator $\mathrm{ADS}^{\mathrm{TM}}$ momentum. The filter was designed at an arbitrary center frequency of $8.04 \mathrm{GHz}$ and 3-dB bandwidth of $40.3 \%$. The filter design was fabricated on standard substrate Arlon CuClad217LX with thickness $h$ of $0.794 \mathrm{~mm}$, dielectric constant $\varepsilon_{r}$ of 2.17, thickness of conductor $t$ of 35 microns, and loss-tangent $\delta$ of 0.0009 . Photograph of the fabricated wideband filter is shown in Fig. 5(a). Magnitude of filter's parameters (in millimeters) are $W_{z o}=2.42, W_{a}=0.2, W_{c l}=0.4, L_{a 1}=1.034, L_{1}$ $=1.5, L_{2}=6.62, L_{3}=0.7, L_{4}=0.99, L_{5}=1.726, L_{6}=2.97, L_{7}=$ $1.09, L_{8}=1.09, S_{1}=0.3575$, and $S_{2}=0.263$.

The measured frequency response of the filter is shown in Fig. 5(b). The measured frequency response shows a very sharp passband skirt, which is centered around $8.04 \mathrm{GHz}$ with a passband insertion-loss that varies between 1.2 to $1.42 \mathrm{~dB}$. The insertion-loss at the filter's center frequency is $1.2 \mathrm{~dB}$ and the passband return-loss is better than $20 \mathrm{~dB}$. The insertion-loss is mainly attributed to conductor and dielectric losses. The four transmission zeros near the lower and upper cutoff frequencies that are located at 6.2, 9.4, 13.6 and $17.4 \mathrm{GHz}$, respectively, contribute toward the filter's sharp selectivity. The 3-dB fractional bandwidth of the filter is $39.8 \%$.

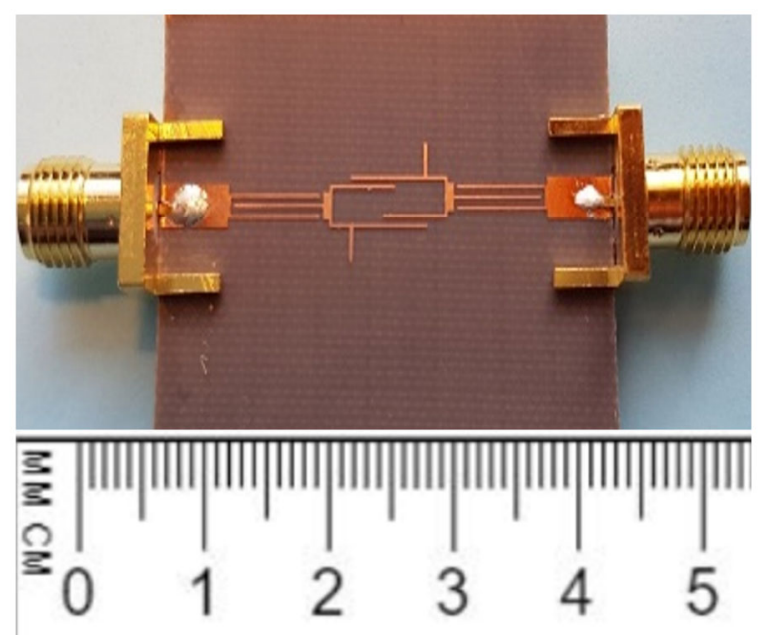

(a)

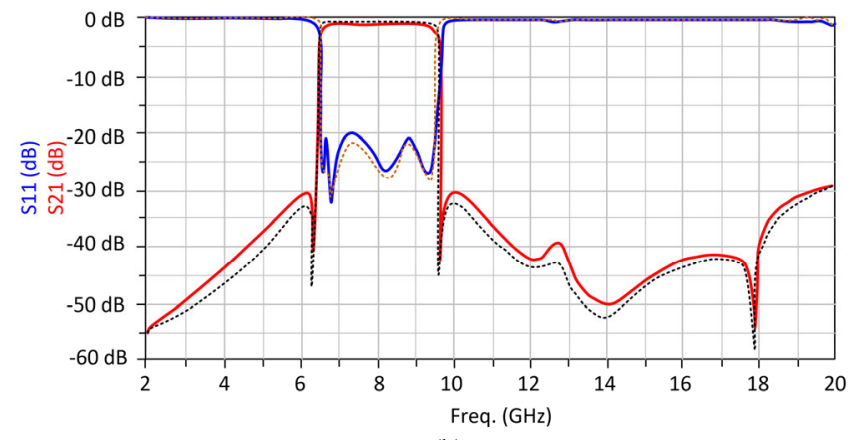

(b) 


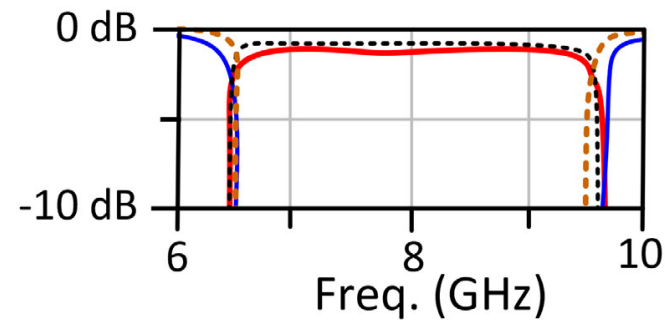

(c)

Fig. 5. (a) Photograph of the fabricated wideband filter, (b) Measured insertionloss and return-loss response of the wideband filter, and (c) close-up of the insertion-loss and return-loss response. (Dot lines are simulation results).

Table I compares the performance of the proposed filter with other planar microstrip wideband filters published recently. Compared to the cited references [1]-[11] the proposed filter has a significantly sharper selectivity, comparable passband insertion-loss, and a significantly wider stopband. In particular, the selectivity factor of the proposed filter of $93 \%$ is much better than that obtained with the high-temperature superconductor bandpass filter whose selectivity is $87.9 \%$ [11]. Although the proposed filter exhibits a return-loss that is better than $20 \mathrm{~dB}$ compared 15.2 by the HTS filter, however its insertion-loss of $1.42 \mathrm{~dB}$ is higher than $0.42 \mathrm{~dB}$ by the HTS filter. The span of the upper stopband of the proposed filter is $10.55 \mathrm{GHz}$ which is substantially larger than $1.17 \mathrm{GHz}$ by the HTS filter. Moreover, the design and fabrication of the proposed filter is simpler, and it does not require any cooling system which will have a major impact on the manufacturing cost.

TABLE I

PERFORMANCE COMPARISON OF THE PROPOSED WIDEBAND BANDPASS FILTER WITH RECENT WORKS

\begin{tabular}{|c|c|c|c|c|c|c|c|}
\hline Ref. & $\begin{array}{l}\text { S.F. } \\
(\%)\end{array}$ & $\begin{array}{c}3-d B \\
F B W \\
(\%)\end{array}$ & $\begin{array}{c}I L \\
(\mathrm{~dB})\end{array}$ & $\begin{array}{c}R L \\
(\mathrm{~dB})\end{array}$ & $\begin{array}{l}\text { Lower } \\
\text { stopband } \\
\text { range } \\
(\mathrm{GHz}) \\
\text { for } \\
\left|\mathrm{S}_{21}\right| \leq-30 \\
\mathrm{~dB}\end{array}$ & $\begin{array}{c}\text { Upper } \\
\text { stopband } \\
\text { range } \\
(\mathrm{GHz}) \\
\text { for } \\
\left|\mathrm{S}_{21}\right| \leq-30 \\
\mathrm{~dB}\end{array}$ & $\begin{array}{l}\text { Size } \\
\lambda_{\mathrm{g}} \times \lambda_{\mathrm{g}}\end{array}$ \\
\hline [1] & 78.4 & 54 & 1.50 & 15 & $\begin{array}{c}2.40- \\
2.70\end{array}$ & $\begin{array}{c}5.40- \\
6.30\end{array}$ & $0.50 \times 0.25$ \\
\hline [2] & 71.0 & 32.9 & 0.95 & 11 & $\begin{array}{c}2.10- \\
2.40\end{array}$ & $\begin{array}{c}4.00- \\
8.00\end{array}$ & $0.32 \times 0.12$ \\
\hline [3] & 78.8 & 28 & 0.91 & 20 & $\begin{array}{c}2.51- \\
2.60\end{array}$ & $\begin{array}{c}3.60- \\
3.65\end{array}$ & $0.55 \times 0.35$ \\
\hline [4] & 70.8 & 35.5 & 1.20 & 14 & $\begin{array}{c}2.00- \\
4.00\end{array}$ & $\begin{array}{c}7.50- \\
8.50\end{array}$ & $2.35 \times 0.77$ \\
\hline [5] & 50.0 & 22 & 1.20 & 22 & $\begin{array}{c}5.50- \\
10.0\end{array}$ & $\begin{array}{c}2.40- \\
5.20\end{array}$ & $0.64 \times 0.57$ \\
\hline [6] & 42.0 & 9.67 & 1.20 & 19 & $\begin{array}{c}0.00- \\
0.29\end{array}$ & $\begin{array}{c}0.37- \\
1.53\end{array}$ & $0.078 \times 0.082$ \\
\hline [7] & 44.9 & 9.05 & 1.20 & 19 & $\begin{array}{c}2.00- \\
3.07\end{array}$ & $\begin{array}{c}3.077- \\
9.76\end{array}$ & $0.078 \times 0.082$ \\
\hline [8] & 62.5 & 26.7 & 0.51 & 21 & $\begin{array}{c}0.00- \\
0.29\end{array}$ & $\begin{array}{c}0.48- \\
1.98\end{array}$ & $0.089 \times 0.10$ \\
\hline [9] & 6.4 & 5.24 & 1.60 & 15 & $\begin{array}{c}1.00- \\
1.81\end{array}$ & - & $0.125 \times 0.085$ \\
\hline [10] & 71.2 & 79.3 & 0.94 & 19 & $\begin{array}{c}0.00- \\
1.44\end{array}$ & $\begin{array}{c}4.56- \\
7.44\end{array}$ & $0.66 \times 0.41$ \\
\hline [11] & 87.9 & 121.4 & 0.42 & 15.2 & $\begin{array}{c}0.00- \\
2.00\end{array}$ & $\begin{array}{c}12.16- \\
13.33\end{array}$ & $1.57 \times 1.18$ \\
\hline $\begin{array}{l}\text { This } \\
\text { work }\end{array}$ & 93.0 & 39.8 & 1.42 & 20 & $\begin{array}{c}0.00- \\
1.40\end{array}$ & $\begin{array}{c}9.45- \\
20.0\end{array}$ & $1.07 \times 0.62$ \\
\hline
\end{tabular}

S.F. $=$ Selectivity Factor $\left(\Delta f_{3 d B} / \Delta f_{30 d B}\right), \Delta f_{3 \mathrm{~dB}}=3 \mathrm{~dB}$ Bandwidth, $\Delta f_{30 \mathrm{~dB}}=30 \mathrm{~dB}$ Bandwidth, $I L=$ Average passband insertion-loss, $R L=$ Return-loss, \& $\mathrm{S}_{21}=$ Insertion-loss

\section{CONCLUSION}

The design and feasibility of a compact quasi-elliptic function wideband bandpass filter exhibiting a significantly wide stopband has been validated with measured results. The filter was fabricated using standard microstrip integrated circuit technology. The measured results show the filter possesses desired characteristics of relatively low-loss, steep skirts for high-selectivity and a very wide stopband. Such filter characteristics are normally achieved with high-temperature superconductors. The proposed filter structure design allows adjustment of the center frequency and upper transmission zeros. The high-selectivity wideband filter is suitable for application such as cognitive radio systems and wireless communication systems that operate in highly interfering environments. The proposed via-free quasi-elliptical wideband filter is relatively simple to design and manufacture using standard PCB technology.

\section{REFERENCES}

[1] P. Cai, Q. Li, "A novel compact wideband filter using three-mode dualring resonator," Int J. RF and Microwave Computer Aided Engineering, Sept. 2018, pp. 1-5.

[2] X. Guan, S. Xie, B. Ren, X. Li, "Compact four-pole wideband bandpass filter with mixed electric and magnetic coupling," Microwave and Optical Technology Letters, Nov. 2019, pp. 1-5.

[3] L. Xu, Z. X. Wang, F. Wei, R. Li, X. T. Zou, "A highly selective balanced wideband bandpass filter based on nested split-ring resonators," Int J. RF and Microwave Computer Aided Engineering, May 2019, pp. 1-7.

[4] L. Yang, F. Xu, J. Qiang, S. Liu, J. Zhan, "A novel wideband bandpass filter based on CSRR-loaded substrate integrated folded waveguide," Int J. RF and Microwave Computer Aided Engineering, January 2020, pp. $1-9$

[5] B. Zhang, C. Yu, Y. Wu, Y. Liu, "Compact wideband filtering microstrip crossover with separated operating frequencies," Microwave and Optical Technology Letters, Feb. 2018, pp. 731-735.

[6] V. K. Killamsetty, B. Mukherjee, "Compact selective bandpass filter with wide stopband for TETRA band applications," IEEE Trans. on Components, Packaging and Manufacturing Technology, Vol. 8, No. 4, April 2018, pp. 653-658.

[7] F. Zhu, W. Hong, J.X. Chen, K. Wu, "Quarter-wavelength steppedimpedance resonator filter with mixed electric and magnetic coupling," IEEE Microwave and Wireless Comp. Letters, Vol. 24, No. 2, Feb. 2014, pp. $90-92$.

[8] V. K. Killamsetty, B. Mukherjee, "Miniaturised highly selective wideband bandpass filter using dual-mode resonators and inter digital capacitors," Electronics Letters, Vol. 53, No. 17, August 2017, pp.12091211 .

[9] B.-F. Zong, G.-M. Wang, J.-G. Liang, C. Zhou, "Compact bandpass filter with two tunable transmission zeros using hybrid resonators," IEEE Microwave and Wireless Comp. Letters, Vol. 25, No. 2, Feb. 2015, pp. 88-90.

[10] Y. Wu, W. Zhang, L. Jiao, Z. Zhuang, Y. Liu, "High performance singleended wideband and balanced bandpass filters loaded with steppedimpedance stubs," IEEE Access, Vol. 5, 2017, pp. 5972-5981.

[11] Z. Long, M. Tian, T. Zhang, M. Qiao, T. Wu, Y. Lan, "High-temperature superconducting multimode dual-ring UWB bandpass filter," IEEE Trans. On Applied Superconductivity, Vol. 30, No. 2, March 2020, 1500204, pp. 1-4. 